\title{
The Effect of Rice Variety and Parboiling on in vivo Glycemic Response
}

\author{
P.M.H.D. Pathiraje, W.M.T. Madhujith ${ }^{1 *}$, A. Chandrasekara ${ }^{2}$ and S.P. Nissanka ${ }^{3}$
}

Department of Food Science and Technology

Faculty of Livestock, Fisheries and Nutrition

Wayamba University of Sri Lanka

\begin{abstract}
Improved and traditional rice varieties grown in Sri Lanka namely, Bg 300, Bg 352, Bg 358, Bg 406, LD 356, Rathkaral, Wedaheenati and Heendikwel were studied for their in vivo glycemic response. Proximate compositions and amylose content of rice were determined according to standard methods and available carbohydrate content was calculated using the difference method. The in vivo glycemic response of selected improved and traditional rice varieties was assessed by determining the glycemic index (GI) using ten healthy subjects. Further, the effect of parboiling of rice on glycemic response was also assessed. The crude protein content was higher in parboiled rice as compared to nonparboiled rice. According to the amylose content, rice varieties studied were classified as intermediate and high amylose rice. The amylose content of Bg 406 was the lowest $(20.18 \% \pm 0.17)$ while Rathkaral showed the highest $(29 \% \pm 0.07)$. The Glycemic index of rice varieties studied ranged from $57 \pm 1$ to $73 \pm 2$. The Wedaheenati variety exhibited the lowest GI while Bg 406 exhibited the highest GI value. Unparboiled Bg 406, LD 356 and parboiled Bg 406 were classified as high GI foods while the rest of the rice varieties studied were categorized as intermediate GI foods. Parboiled rice brought about a reduction in glycemic response in healthy subjects. The maximum reduction of $10 \%$ in glycemic index upon parboiling was observed with Bg 352. The traditional rice produced significantly lower $(p<0.05)$ postprandial glycemic effect than did the improved rice. By their low post-prandial glycemic response they could be potentially useful in low GI diets.
\end{abstract}

Key words: Aamylose, Glycemic index (GI), Parboiling, Rice.

\section{INTRODUCTION}

Rice is the dietary staple of the people of Sri Lanka. It comprises of $75-80 \%$ carbohydrates as starch. The fact that higher proportion of rice starch is included in the Sri Lankan diet signifies the need for investigating the bioavailability and metabolic responses of rice. There is a considerable variation in bioavailability of carbohydrates among different foods though a higher percentage of carbohydrates in most human diet is digested and absorbed in the small intestine. It has been suggested that diets containing large amounts of rapidly digestible carbohydrates may be detrimental to health as they elevate glucose concentration in blood, which is crucial for diabetics (Jenkins et al. 1988). Glycemic index (GI) has been developed for the systematic classification of foods containing carbohydrates, which provides a means of quantitatively comparing the blood glucose responses following ingestion of equivalent

1* To whom correspondence should be addressed to: madujith@yahoo.com

Department of Food Science and Technology, Faculty of Agriculture, University of Peradeniya, Sri Lanka

${ }^{2}$ Faculty of Health Medicine Nursing and Behavioural Sciences, Deakin University, Geelong, Australia

${ }^{3}$ Department of Crop Science, Faculty of Agriculture, University of Peradeniya, Sri Lanka 
amounts of digestible carbohydrates from different foods. It has been suggested that low GI foods have beneficial effects in the management of diabetes and hyperlipidemia and high GI foods are crucial as they rapidly elevate glucose concentration in blood (Brand-Miller et al., 2002; Mosdol et al., 2007).

Studies have shown that the digestibility of starch is partly attributed to the inherent properties of starch including crystallinity, granular structure and amylose: amylopectin ratio. Therefore, the type of crop and variety play an important role in determining the rate of digestion of their starch, hence the GI. It has become difficult to classify rice cultivated in the world as high or low GI food based on the results around the world. Glycemic Index of rice has been established as low as 59 to as high as 109 (Miller et al., 1992; Frei et al., 2003). Some of the commercial rice varieties available in Sri Lanka have shown GI values ranging from 55 to 73 (Hettiarachchi et al., 2000) with majority has been classified as medium GI rice.

Sri Lanka produces a range of rice varieties which have been classified according to their grain characteristics. Based on the colour of pericarp, rice is classified as white and red rice whereas it is classified as long $(n a d u)$ and short (samba) rice based on the grain size. Although there is no scientific evidence, people believe that red rice is better in reducing blood glucose levels in diabetics. In this context, the present study was conducted to study the glycemic properties of different types of rice available in Sri Lanka.

\section{MATERIALS AND METHODS}

Rough rice samples of improved (Bg 300, Bg 352, Bg 358, Bg 406 and LD 356) and traditional rice varieties (Wedaheenati, Rathkaral and Heendikwel) were obtained from Rice Research and Development Institute, Bathalagoda and Department of Crop Science, Faculty of Agriculture, University of Peradeniya, respectively. Samples of rice were cleaned of extraneous material and dehulled followed by double pass whitening in a laboratory mill (model PM 500). The samples of parboiled rice were prepared by, steeping rice in excess water at room temperature for $8 \mathrm{~h}$ followed by steaming until the grains in the top began to split. Steamed rice was then sun dried until the grain moisture content reached $12-14 \%$.

\section{Chemical analysis}

The cooked and dried rice were ground to a fine powder in an electric blender and analyzed for proximate composition as per AOAC methods (2000). The available carbohydrate content of each rice variety was calculated using the difference method. Amylose content was determined according to the method outlined by ISO, 6647 (1987) with minor modifications.

\section{In vivo glycemic response}

A group of 10 volunteers from both sexes aged between 25 and 45 years with a normal BMI and apparently healthy with no family history of diabetes, impaired glucose tolerance or obesity were selected for the study. The subjects were not on medication for any disease and all were non-smokers. Before being admitted to the study, subjects were given a detailed written and oral explanation of the different procedures involved in the study and written informed consent was obtained from all subjects. Cooking of rice was carried out according to preset conditions adding similar quantity of water and rice and similar duration of cooking for a particular variety of rice. After 10 to $12 \mathrm{~h}$ overnight fast, subjects were given cooked 
rice portions containing $50 \mathrm{~g}$ of available carbohydrates, which were consumed over 10 to 15 min. Test food was given with $5 \mathrm{~g}$ of chilli paste and $250 \mathrm{~mL}$ of water. Finger prick blood samples were obtained at 0 (fasting), 15, 30, 45, 60, 90, 120 min after the ingestion of the test food. The concentration of glucose in blood was measured using a glucometer. Glycemic response to the test foods was compared with the response to the reference food. Reference food was glucose that was prepared by dissolving $50 \mathrm{~g}$ of glucose in $250 \mathrm{~mL}$ of water. For each rice type, the incremental area under the curve (AUC) was calculated and compared with the average AUC for the glucose in that particular subject. Any area under the base line was ignored. GI was calculated using the following equation and presented as the mean \pm SEM.

$$
G I=\frac{\text { Incremental AUC for rice }}{\text { Incremental AUC for glucose }} \times 100
$$

\section{Statistical analysis}

Data obtained from each subject were fitted into a completely randomized design (CRD) and subjected to analysis of variance and multiple comparisons to determine statistical differences among varieties at a significance level of 0.05 .

\section{RESULTS AND DISCUSSION}

Weight of cooked rice portions containing $50 \mathrm{~g}$ of available carbohydrate was lowest in unparboiled $\mathrm{Bg} 406$ (143 g) and highest in $\mathrm{Bg} 300$ (195 g) (Table 1). All rice types contained approximately the same quantity of crude protein, crude fiber, crude fat and ash. The rice varieties with red pericarp contained significantly $(\mathrm{p}<0.05)$ higher crude fiber content than did the rice with white pericarp. The results further showed that the crude protein content in parboiled rice was relatively higher as compared to their unparboiled counterparts.

The postprandial blood glucose level (Fig. 1) reached a peak 30 min after the ingestion of unparboiled rice. Among the rice varieties, the maximum peak was observed with Rathkaral whereas $\mathrm{Bg} 406$ exhibited the lowest peak in blood glucose response curve. The area under the curve for blood glucose response to all rice varieties were significantly $(p<0.05)$ lower than that observed for pure glucose.

Table 1. Proximate composition and weight of cooked rice portions equivalent to $50 \mathrm{~g}$ available carbohydrate

\begin{tabular}{lcccccc}
\hline Variety & $\begin{array}{c}\text { Weight of } \\
\text { portion (g) }\end{array}$ & Moisture (g) & $\begin{array}{c}\text { Crude } \\
\text { protein (g) }\end{array}$ & $\begin{array}{c}\text { Crude } \\
\text { fibre (g) }\end{array}$ & Ash (g) & $\begin{array}{c}\text { Crude } \\
\text { fat (g) }\end{array}$ \\
\hline Bg 300 UPB & 195 & 141.74 & 2.50 & 0.38 & 0.33 & 0.20 \\
Bg 352 UPB & 162 & 108.74 & 2.14 & 0.38 & 0.26 & 0.23 \\
Bg 358 UPB & 150 & 96.26 & 2.21 & 0.43 & 0.38 & 0.31 \\
Bg 406 UPB & 143 & 90.03 & 2.23 & 0.55 & 0.34 & 0.26 \\
Bg 356 UPB & 149 & 94.79 & 2.67 & 0.52 & 0.29 & 0.28 \\
Rathkaral UPB & 181 & 127.22 & 2.76 & 0.52 & 0.26 & 0.31 \\
Wedaheenati UPB & 191 & 136.92 & 3.16 & 0.59 & 0.28 & 0.34 \\
Heendikwel UPB & 173 & 119.58 & 2.69 & 0.52 & 0.34 & 0.32 \\
Bg 352 PB & 159 & 104.46 & 3.50 & 0.39 & 0.26 & 0.32 \\
Bg 358 PB & 147 & 92.86 & 3.24 & 0.42 & 0.29 & 0.38 \\
Bg 406 PB & 146 & 92.11 & 3.59 & 0.57 & 0.29 & 0.32 \\
Bg 356 PB & 143 & 89.86 & 2.59 & 0.50 & 0.26 & 0.33 \\
\hline
\end{tabular}


PB - parboiled, UPB- unparboiled

The peak blood glucose response was observed $45 \mathrm{~min}$ after the ingestion of parboiled rice. This reveals that the parboiled rice takes longer time to be digested in healthy subjects compared to the unparboiled rice (Fig. 1. b through e).

As shown in Table 2, the GI of rice varieties investigated in the present study ranged from $57 \pm 1$ to $73 \pm 2$. Result showed that there were significant differences in the glycemic responses of healthy individuals to different varieties of rice. Traditional rice variety Wedaheenati exhibited the lowest, whereas $\mathrm{Bg} 406$ exhibited the highest GI. According to the categorization of carbohydrate rich foods based on GI, the rice varieties studied can be classified as high and medium GI (Table 2). Parboiled and unparboiled BG 406 and unparboiled LD 356 fell into high GI foods whereas all other rice varieties studied fell into medium GI rice. Both parboiled and unparboiled $\mathrm{Bg} 406$ samples yielded low GI values. Unparboiled $\mathrm{Bg}$ 356, 352, and 358, and both parboiled and unparboiled $\mathrm{B} g 406$ exhibited significantly $(\mathrm{p}<0.05)$ higher GI values among the rice varieties studied.

(a)

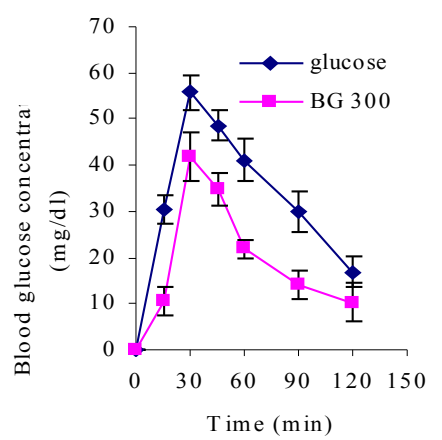

(c)

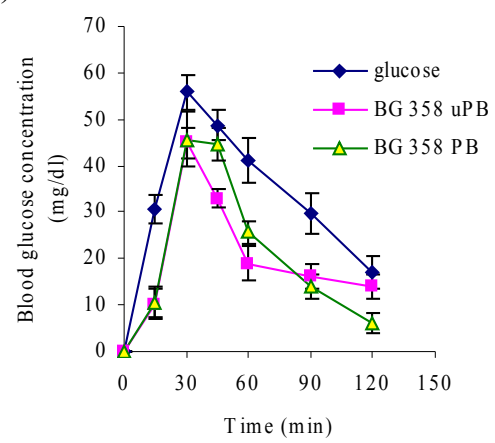

(b)

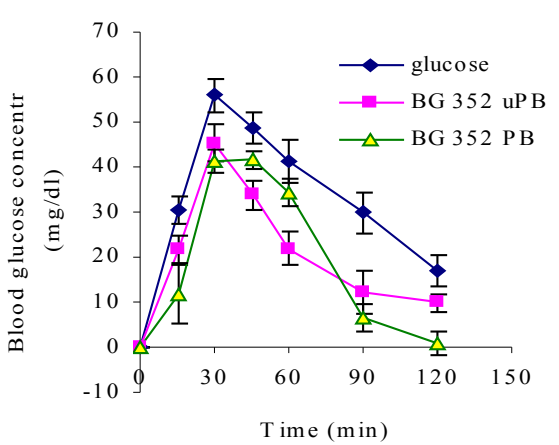

(d)

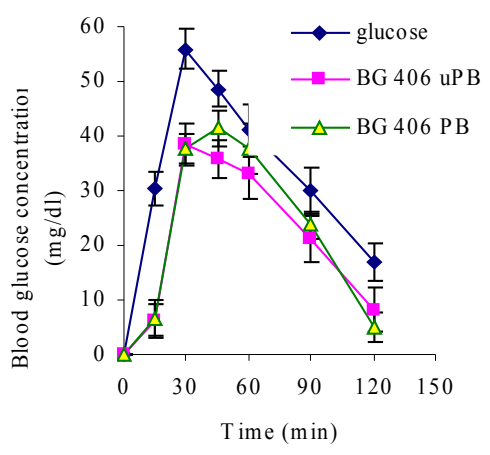


(e)

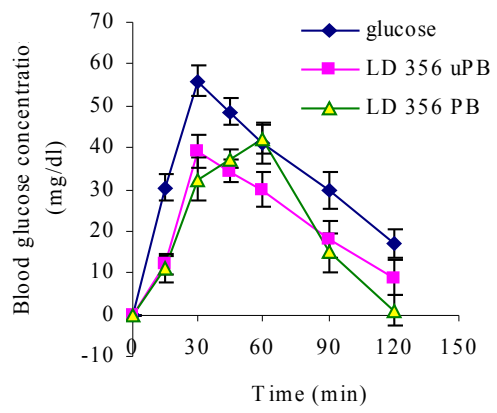

(g)

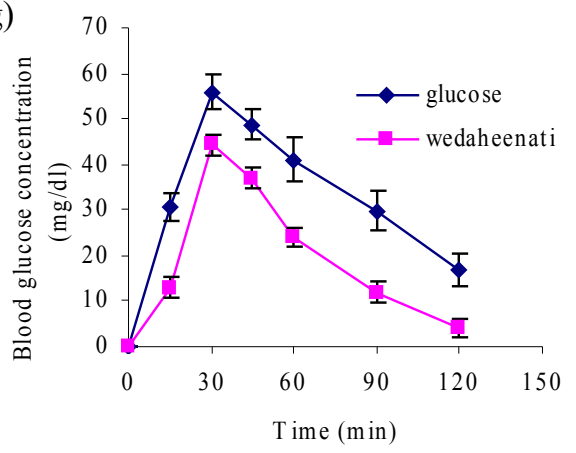

(f)

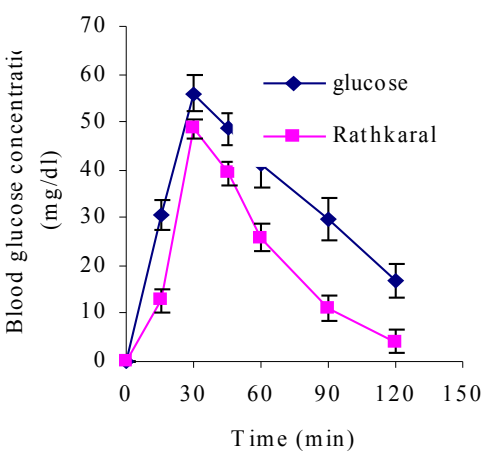

(h)

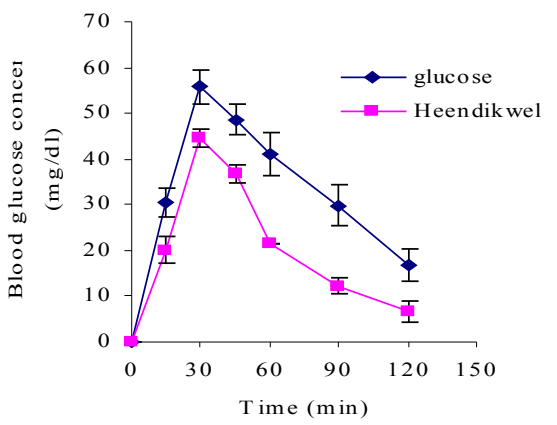

Fig. 1. Postprandial glucose responses to different improved and traditional rice varieties in healthy subjects (a) $\mathrm{Bg} 300$, (b) $\mathrm{Bg} \mathrm{352}$, (c) $\mathrm{Bg} 358$ (d) $\mathrm{Bg} 406$ (e) LD 356 (f) Rathkaral (g) Wedaheenati and (h) Heendikwel. PB - parboiled and uPB - unparboiled.

Diets that result in gradual and slow increase in postprandial blood glucose concentration are gaining increased attention as they can potentially reduce the risk of diseases related to impaired glucose metabolism (Brigheuti et al., 2006). Therefore, majority of the rice varieties studied which fall into medium GI foods would be beneficial to balance blood glucose level in diabetics when they are incorporated in their diets.

Results of GI studies around the world reveal that GI of rice varies widely among different cultivars or varieties. Miller et al., (1992) noticed GI values ranging from $64 \pm 9$ to $93 \pm 11$ for rice. GI values ranging from 68 to 109 were observed by Frei et al. (2003). These results revealed that some rice varieties produce larger glycemic response as high as the glycemic response produced by glucose or white bread. However, as compared to the above results, the varieties considered in the present study showed comparatively low GI values.

Starches with high amylose content are less susceptible to hydrolysis by $\alpha$ - amylase mainly because amylose has a larger molecule size than amylopectin and does not have a branched, but a linear structure (Fitzgerald, 2004). The amylose content of the rice varieties investigated in the present study varied from $21.50 \% \pm 0.30$ to $29.5 \% \pm 0.36$ (Table 2). There was a significant $(\mathrm{p}<0.05)$ negative correlation between GI and amylose content. This is in accordance with the findings of Miller et al. (1992). They have also reported that the rice with higher amylose content gives a significantly lower GI than did the rice with low 
amylose content. However, others have reported a non significant correlation between amylose content and digestibility of starch (Frei et al., 2003).

Rice varieties cultivated in Sri Lanka are generally classified as white and red rice based on the colour of the pericarp. The unparboiled red rice is popular among patients with diabetes in Sri Lanka. However, the results of the present study do not support this belief. ANOVA showed that rice with red pericarp does not give significantly low GI, and poor correlation between grain colour and GI was exhibited in the correlation analysis. In this study, red rice varieties Heendikwel, Rathkaral and Wedaheenati gave comparatively lower GI of 62, 60 and 57 , respectively.

Table 2. Glycemic index and amylose content (\% dry wt basis) of different rice varieties

\begin{tabular}{lccl}
\hline Variety & $\begin{array}{c}\text { Amylose content } \\
(\mathbf{\%})\end{array}$ & $\begin{array}{c}\text { GI } \pm \text { SEM } \\
(\mathbf{n}=\mathbf{1 0})\end{array}$ & GI category \\
\hline $\mathrm{Bg} 300$ UPB & $27.1 \pm 0.16^{\mathrm{c}}$ & $61 \pm 3^{\mathrm{a}}$ & Medium \\
$\mathrm{Bg} 352$ UPB & $27.0 \pm 0.17^{\mathrm{c}}$ & $67 \pm 3^{\mathrm{b}}$ & Medium \\
$\mathrm{Bg} 358$ UPB & $29.0 \pm 0.52^{\mathrm{d}}$ & $67 \pm 6^{\mathrm{b}}$ & Medium \\
$\mathrm{Bg}$ 406 UPB & $24.5 \pm 0.70^{\mathrm{b}}$ & $73 \pm 2^{\mathrm{b}}$ & High \\
LD 356 UPB & $21.5 \pm 0.30^{\mathrm{a}}$ & $70 \pm 2^{\mathrm{b}}$ & High \\
Rathkaral UPB & $29.5 \pm 0.06^{\mathrm{d}}$ & $60 \pm 2^{\mathrm{a}}$ & Medium \\
Wedaheenati UPB & $29.0 \pm 0.36^{\mathrm{d}}$ & $57 \pm 1^{\mathrm{a}}$ & Medium \\
Heendikwel UPB & $27.7 \pm 0.19^{\mathrm{c}}$ & $62 \pm 2^{\mathrm{a}}$ & Medium \\
$\mathrm{Bg}$ 352 PB & n.d. & $60 \pm 2^{\mathrm{a}}$ & Medium \\
$\mathrm{Bg} 358$ PB & n.d. & $62 \pm 2^{\mathrm{a}}$ & Medium \\
LD 356 PB & n.d. & $64 \pm 5^{\mathrm{a}}$ & Medium \\
$\mathrm{Bg}$ 406 PB & n.d. & $71 \pm 2^{\mathrm{b}}$ & High \\
\hline
\end{tabular}

Means within the same column bearing different superscripts are significantly $(\mathrm{p}<0.05)$ different. PB -parboiled, UPB- unparboiled, n.d. - not determined.

$\mathrm{Bg} 406$ and LD 356 showed significantly $(\mathrm{p}<0.05)$ higher GI values than $\mathrm{Bg} 300, \mathrm{Bg} 352$ and Bg 358 although they have a red pericarp. These observations indicate that the GI of a rice variety could not be predicted based on its grain colour. However, Perera and Jansz (2000) have suggested that parboiling and red pigment together can slow down the enzymatic hydrolysis of starch.

The results revealed that parboiling tends to reduce the glycemic response in healthy subjects (Fig. 2). Results of ANOVA exhibited that a significant $(\mathrm{P}<0.05)$ difference exists between the observed GI of parboiled and unparboiled rice. The maximum reduction in GI of $10 \%$ upon parboiling was observed in $\mathrm{B} g$ 352. The retrogradation of starch brought about by parboiling of rice could be attributed to the reduced GI in parboiled rice compared to their unparboiled counterparts. Moreover, the presence of higher proportions of amylose in the starch may promote the retrogradation of starch in this rice variety. In addition, protein was found to reduce the access of enzymes to starch through physical encapsulation and starchprotein interactions are reported to reduce the digestibility of starch in wheat bread (Jenkins et al., 1987). However, the protein content of wheat bread is higher than that of rice. With respect to the protein content of rice varieties used in the present study, parboiled rice 
contained higher protein content than unparboiled rice. According to these results, it could be further suggested that the observed low GI of parboiled rice may perhaps be due to the high protein content and increased physical hardness of the grains for digestion.

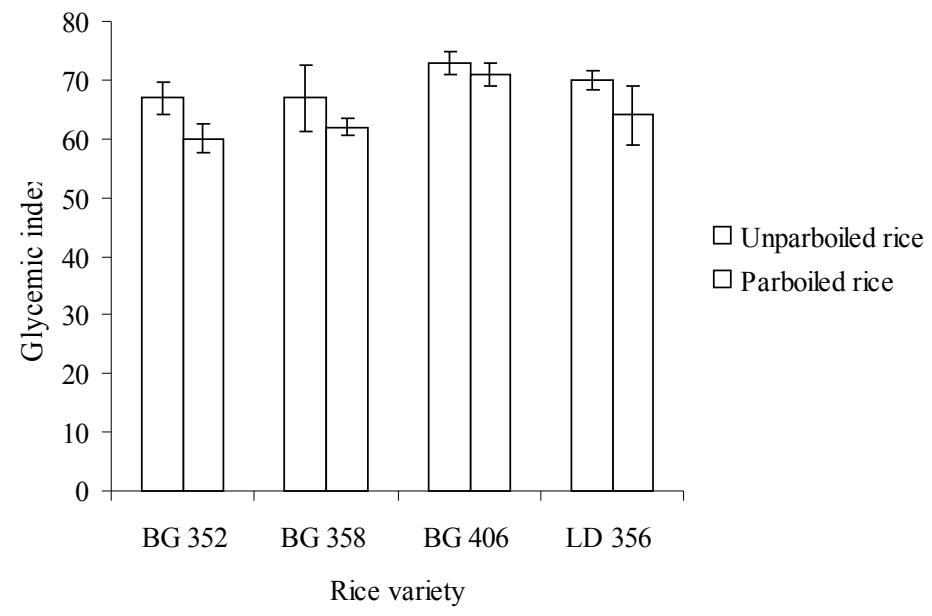

Fig. 2. Effect of parboiling on glycemic index of selected improved rice varieties

In addition to the improved rice varieties grown in Sri Lanka, there are traditional rice varieties which fueled the diet of Sri Lankan people in the past. The extinction of traditional rice has begun with the evolution of improved varieties and the latter became more popular due to improved characteristics such as resistance to pests and diseases, higher yields and better cooking and eating characteristics. However, consumers still believe that traditional rice has better nutritional properties and they tend to keep them under satire condition for a long time. Among the traditional rice varieties, Rathkaral, Wedaheenaty and Heendikwel are three varieties commonly consumed by Sri Lankans. Results revealed that, traditional rice produced a significantly $(\mathrm{P}<0.05)$ lower postprandial glycemic effect than did the improved varieties. The lowest GI among all the rice varieties studied in the present study was observed in Wedaheenati. Moreover, the parboiled rice of improved varieties except $\mathrm{Bg} 352$, showed higher GI compared to unparboiled rice of traditional varieties. According to these results, it could be suggested that traditional rice possibly possess beneficial effects for health since they tend to be digested slowly compared to improved rice.

\section{CONCLUSIONS}

The amylose content of rice shows a strong negative correlation with the GI value of rice. Parboiling generally reduces the GI of most of the rice varieties. The traditional rice varieties studied exhibit lower GI than improved rice vareties. The colour of the grain pericarp does not appear as a good indicator in predicting its glycemic index. Based on the results of the present study, it can be concluded the GI value varies significantly with the rice variety Moreover, the GI of the rice varieties investigated in the present study ranged in the moderate and high GI foods. These varying results emphasize the need to carry out glycemic index testing for other rice varieties available for consumption in Sri Lanka. 


\section{ACKNOWLEDGEMENTS}

The authors wish to thank Wayamba University of Sri Lanka, Kuliyapitiya for the financial support and the Rice Research and Development Institute, Bathalagoda for providing material for the study.

\section{REFERENCES}

AOAC (2000). Official Methods of Analysis. AOAC International, Gaithersburg, Maryland, USA. pp. 1-38.

Brand-Miller, J.C., Holt, S.H.A., Pawlak, D.B. and Mcmillan, J. (2002). Glycemic index and obesity. Am.J, Clin. Nutr. 76 (suppl), 281S - 285S.

Brigheuti, F., Benimi, L., Del Rio, D., Casiraghi. C. Pellegrini, N. and Scazzian, F. (2006). Colonic fermentation of indigestible carbohydrates contribute to the second meal effect. Am. J. Clin Nutr. 83, 817 - 822.

Fitzgerald, M. (2004). Starch. In: Champagne, E.T. (Ed). Rice Chemistry and Technology, Association of Cereal Chemists, USA. pp. 109-141.

Frei, M., Siddhuraju P. and Becker, K. (2003). Studies on the in vitro starch digestibility and the glycemic index of six different indigenous rice cultivars from the Phillipines. Food Chem. 83, 395 - 402 .

Hettiarachchi, P., Jiffry, M.T.M., Jansz, E.R., Wickramasinghe, A.R., Fernando, D.J.S. (2001). Glycemic indices of different varieties of rice grown in Sri Lanka. Ceylon Med. J. 46(1), $11-14$.

ISO (1987). Rice: Determination of amylose content. Method 6647. International organization for Standardization, Geneva.

Jenkins, D.J.A., Thorne, M.J., Wolver, T.M.S., Jenkins, A.L., Rao, A.V., and Thompson, L.U. (1987). The effect of starch protein interactions in wheat on glycemic response and rate of in vitro starch digestion. Am. J. Clin. Nutr. 45, 946-95.

Jenkins, D.J.A., Wolver, T.M.S., Buckley, G., Lam, K.Y., Giudici, S., Kalmusky, J., Jenkins. A.L., pattern, R.L., Bird, J., Wong, G.S. and Josse, R.G. (1988). Low glycemic index starchy foods in the diabetic diet. Am. J. Clin. Nutr. 48, 284 - 254.

Jenkins, D.J., Wolver, T.M., Taylor, R.H., Barker, H., Fielden, H., Baldwin, J.M., Bowlin, A.C., Newman, H.C., Jenkins, A.Z. and Goff. D.V. (1981). Glycemic index of foods: a physiological basis for carbohydrate exchange. Am. J. Clin Nutr. 34(3), 362 - 366.

Miller, J.B., Pang, E. and Brammal, L., (1992). Rice: a high or low glycemic index food. Am. J. Clin Nutr. 56, 1034 - 1036.

Mosdol, A., Witte, D.R., Frost, G., Marmot, M.G. and Barnner, E.J., (2007). Dietary glycemic index and glycemic load are associated with high density - lipoprotein cholesterol at baseline but not with increased risk of diabetes in the white hall II study. Am. J. Clin. Nutr. 86, 988 - 994. 
Perera, A.S., and Jansz, E.R. (2000). Preliminary investigations on the red pigment in rice and its effect on glucose release from rice starch. J. Natn. Sci. Foundation Sri Lanka 28(3), 185-192.

Wildman, R.E.C. and Medeiros, D.M. (2000). Carbohydrates. In: Advanced Human Nutrition, CRC Press, UK. pp. 79-98. 